\title{
ALMOST SOUSLIN KUREPA TREES
}

\author{
MOHAMMAD GOLSHANI
}

(Communicated by Julia Knight)

\begin{abstract}
We show that the existence of an almost Souslin Kurepa tree is consistent with $Z F C$. We also prove their existence in $L$. These results answer two questions from a paper by Zakrzewski.
\end{abstract}

\section{INTRODUCTION}

The theory of trees forms a significant and highly interesting part of set theory. In this paper we study $\omega_{1}$-trees and prove some consistency results concerning them. Let $T$ be a normal $\omega_{1}$-tree. Let's recall that:

- $T$ is an Aronszajn tree if it has no branches.

- $T$ is a Kurepa tree if it has at least $\omega_{2}$-many branches.

- $T$ is a Souslin tree if it has no uncountable antichains (and hence no branches).

- $T$ is an almost Souslin tree if for any antichain $X \subseteq T$, the set $S_{X}=\{h t(x)$ : $x \in X\}$ is not stationary (see [1], [16]).

- $T$ is regressive if for any limit ordinal $\alpha<\omega_{1}$, there is a function $f: T_{\alpha} \rightarrow$ $T_{<\alpha}$ such that for any $x \in T_{\alpha}, f(x)<_{T} x$, and for any $x \neq y$ in $T_{\alpha}$, at least one of $f(x)$ or $f(y)$ is above the meet of $x$ and $y$ (see [8]).

Intuitively a Kurepa tree is very thick. On the other hand, a Souslin tree is very thin, and obviously no Kurepa tree is a Souslin tree. We can think of an almost Souslin tree as a fairly thin tree. The following are well known:

- There is an Aronszajn tree (Aronszajn; see [3] for proof).

- It is consistent with $Z F C$ that a Souslin tree exists (Jech [2], Tennenbaum [13]).

- $V=L$ implies the existence of a Souslin tree (Jensen [5]; see also [6]).

- It is consistent with $Z F C+\neg C H$ to assume there is no Souslin tree (Solovay and Tennenbaum [11]).

- It is consistent with $Z F C+G C H$ to assume there is no Souslin tree (Jensen 7]; see 9] for proof).

- It is consistent with $Z F C$ that a Kurepa tree exists (Stewart [12]; see [3] for proof).

Received by the editors July 12, 2011 and, in revised form, August 13, 2011; August 17, 2011; and September 11, 2011.

2010 Mathematics Subject Classification. Primary 03E35.

The author would like to thank the School of Mathematics, Institute for Research in Fundamental Sciences (IPM), for their support during the preparation of this paper. He also wishes to thank Dr. E. Eslami and Dr. Sh. Mohsenipour for their inspiration and encouragement. 
- $V=L$ implies the existence of a Kurepa tree (Solovay; see [3] for proof).

- It is consistent, relative to the existence of an inaccessible cardinal, that there is no Kurepa tree (Silver [10]).

For more details on trees, we refer the reader to the articles [3] and [14. The following example shows that almost Souslin trees exist in $Z F C$.

Example 1.1. Let $T=\left\{t \epsilon^{<\omega_{1}} 2: \operatorname{Supp}(t)\right.$ is finite $\}$. Then it is easily seen that $T$ is an almost Souslin tree with $\omega_{1}$-many branches and with no Aronszajn subtrees (see also [14, Theorem 4.1).

Now, in [16, Zakrzewski asked the following questions:

Question 1.2. Is the existence of an almost Souslin Kurepa tree consistent with $Z F C$ ?

Question 1.3. Does the axiom of constructibility guarantee the existence of an almost Souslin Kurepa tree?

In this paper, we answer both of these questions positively. In section 2 we answer Question 1.2 by building a model of $Z F C$ in which an almost Souslin Kurepa tree exists, and in section 3 we answer Question 1.3 by showing that the existence of an $\left(\omega_{1}, 1\right)$-morass implies the existence of an almost Souslin Kurepa tree.

\section{Forcing an Almost Souslin Kurepa tree}

In this section we answer Question 1.2. In fact we will prove something stronger, which also extends some results from [8].

Theorem 2.1. Assume GCH. Then there exists a cardinal-preserving generic extension of $V$ in which an almost Souslin regressive Kurepa tree exists.

Proof. Let $\kappa \geq \omega_{2}$. We produce a cardinal-preserving generic extension of $V$ which contains an almost Souslin regressive Kurepa tree with $\kappa$-many branches. First we define a forcing notion $\mathbb{P}$ which adds a regressive Kurepa tree with $\kappa$-many branches. This forcing is essentially the forcing notion of [8]. Conditions in $\mathbb{P}$ are of the form $p=\left\langle T_{p}, \leq_{p}, g_{p}, f_{p}\right\rangle$ where:

(1) $T_{p} \subseteq \omega_{1}$ is countable;

(2) $\left\langle T_{p}, \leq_{p}\right\rangle$ is a normal $\left(\alpha_{p}+1\right)$-tree, where $\alpha_{p}$ is an ordinal less than $\omega_{1}$;

(3) $g_{p}$ is a bijection from a subset of $\kappa$ onto $\left(T_{p}\right)_{\alpha_{p}}$, the $\alpha_{p}$-th level of $T_{p}$;

(4) $f_{p}: T_{p, \text { lim }} \rightarrow T_{p}$, where $T_{p, \text { lim }}=\left\{x \in T_{p}: h t(x)\right.$ is a limit ordinal $\}$;

(5) for all $x \in T_{p, l i m}, f_{p}(x)<_{p} x$;

(6) for each $x \neq y$ in $T_{p, l i m}$, if $h t(x)=h t(y)$, then at least one of $f_{p}(x)$ or $f_{p}(y)$ is above the meet of $x$ and $y$.

The order relation on $\mathbb{P}$ is defined by $p \leq q(p$ is an extension of $q)$ iff:

(1) $\left\langle T_{p}, \leq_{p}\right\rangle$ end extends $\left\langle T_{q}, \leq_{q}\right\rangle$,

(2) domg $_{p} \supseteq$ domg $_{q}$,

(3) for all $\alpha \in \operatorname{domg}_{q}, g_{p}(\alpha) \geq_{p} g_{q}(\alpha)$,

(4) $f_{p} \supseteq f_{q}$.

The following lemma can be proved easily (see also [8], Theorem 5). 
Lemma 2.2. (a) Let $p \in \mathbb{P}$ and $\alpha<\kappa$. Then there exists $q \leq p$ such that $\alpha \in$ domg $g_{q}$. Furthermore $q$ can be chosen so that $\alpha_{q}=\alpha_{p}+1$ and domg $g_{q}=d o m g_{p} \cup\{\alpha\}$.

(b) Let $\left\langle p_{n}: n\langle\omega\rangle\right.$ be a descending sequence of conditions in $\mathbb{P}$. Then there exists $q \in \mathbb{P}$ which extends all of the $p_{n}$ 's. Furthermore $q$ can be chosen so that $\alpha_{q}=\sup _{n \in \omega} \alpha_{p_{n}}$ and $\operatorname{dom}\left(g_{q}\right)=\bigcup_{n<\omega} \operatorname{dom}\left(g_{p_{n}}\right)$.

(c) $\mathbb{P}$ satisfies the $\omega_{2}-$ c.c.

It follows from the above lemma that $\mathbb{P}$ is a cardinal-preserving forcing notion. Let $G$ be $\mathbb{P}$-generic over $V$. Let

- $T=\bigcup_{p \in G} T_{p}$,

- $\leq_{T}=\bigcup_{p \in G} \leq_{p}$,

- $f=\bigcup_{p \in G} f_{p}: T_{\text {lim }} \rightarrow T$, where $T_{\text {lim }}=\{x \in T: h t(x)$ is a limit ordinal $\}$.

It is easy to show that $\left\langle T, \leq_{T}\right\rangle$ is a normal regressive $\omega_{1}$-tree.

Lemma 2.3. $\left\langle T, \leq_{T}\right\rangle$ has $\kappa-$ many branches; in particular, it is a Kurepa tree.

Proof. The lemma follows easily from the following facts:

(1) For each $\xi<\kappa,\left\{g_{p}(\xi): p \in G, \xi \in \operatorname{dom}\left(g_{p}\right)\right\}$ determines a branch $b_{\xi}$ of $T$.

(2) For $\xi \neq \zeta$ in $\kappa, b_{\xi} \neq b_{\zeta}$.

Let $S=\left\{\alpha_{p}: p \in G\right.$, domg $\left._{p}=\bigcup\left\{d o m g_{q}: q \in G, \alpha_{q}<\alpha_{p}\right\}\right\}$.

Lemma 2.4. $S$ is a stationary subset of $\omega_{1}$.

Proof. Let $\dot{S}$ be a $\mathbb{P}$-name for $S$. Let $p \in \mathbb{P}$ and $\dot{C}$ be a $\mathbb{P}$-name such that

$$
p \|-\left\ulcorner\dot{C} \text { is a club subset of } \omega_{1}\right\urcorner \text {. }
$$

We find $q \leq p$ which forces $\dot{S} \cap \dot{C} \neq \emptyset$. Define by induction two sequences $\left\langle p_{n}: n<\omega\right\rangle$ of conditions in $\mathbb{P}$ and $\left\langle\beta_{n}: n<\omega\right\rangle$ of countable ordinals such that:

- $p_{0}=p$,

- $p_{n+1} \leq p_{n}$,

- $\alpha_{p_{n}}<\beta_{n}<\alpha_{p_{n+1}}$,

- $p_{n+1} \|-\left\ulcorner\beta_{n} \in \dot{C}\right\urcorner$.

By Lemma 2.2(b), there is $q \in \mathbb{P}$ such that $q$ extends $p_{n}$ 's, $n<\omega$, and such that $\alpha_{q}=\sup _{n \in \omega} \alpha_{p_{n}}$ and $\operatorname{dom}\left(g_{q}\right)=\bigcup_{n<\omega} \operatorname{dom}\left(g_{p_{n}}\right)$. Then it is easily seen that $q \|-\left\ulcorner\alpha_{q} \in \dot{S} \cap \dot{C}\right\urcorner$.

Working in $V[G]$, let $\mathbb{Q}$ be the usual forcing notion for adding a club subset to $S$. Thus conditions in $\mathbb{Q}$ are closed bounded subsets of $S$ ordered by end extension. Let $H$ be $\mathbb{Q}$-generic over $V[G]$. The following is well known (see [4, Theorem 23.8).

Lemma 2.5. (a) $\mathbb{Q}$ is $\omega_{1}$-distributive,

(b) $\mathbb{Q}$ satisfies the $\omega_{2}-$ c.c.,

(c) $C=\bigcup H \subseteq S$ is a club subset of $\omega_{1}$.

It follows that $\mathbb{Q}$ is a cardinal-preserving forcing notion, and hence $\left\langle T, \leq_{T}\right\rangle$ remains a regressive Kurepa tree with $\kappa$-many branches in $V[G][H]$. We show that in $V[G][H],\left\langle T, \leq_{T}\right\rangle$ is also almost Souslin.

Lemma 2.6. In $V[G][H],\left\langle T, \leq_{T}\right\rangle$ is almost Souslin. 
Proof. Suppose not. Let $Z \subseteq T$ be an antichain of $T$ such that $S_{Z}=\{h t(x): x \in Z\}$ is stationary in $\omega_{1}$. We may further suppose that for $x \neq y$ in $Z, h t(x) \neq h t(y)$ and that $S_{Z} \subseteq C$.

First we define a map $h$ on $T \mid C=\{x \in T: h t(x) \in C\}$ as follows: Let $\alpha \in C$ and $x \in T_{\alpha}$. Pick $p \in G$ such that $\alpha=\alpha_{p}$. Then $x=g_{p}(\xi)$, for some $\xi \in d o m g_{p}$. Let $h(x)=g_{q}(\xi)$, where $q \in G$ is such that $\alpha_{q}$ is minimal with $\xi \in d o m g_{q}$. Note that $\alpha_{q}<\alpha_{p}$ and $h(x)<_{T} x$ (as $C \subseteq S$ ).

The map $h t(x) \mapsto h t(h(x))$ is well-defined and regressive on $S_{Z}$; hence by Fodor's lemma there is $Y \subseteq Z$ and an ordinal $\gamma<\omega_{1}$ such that $S_{Y}$ is stationary and for all $x \in Y, h t(h(x))=\gamma$. Since $T_{\gamma}$ is countable, we can find $X \subseteq Y$ and $t \in T$ such that $S_{X}$ is stationary, and for all $x \in X, h(x)=t$. Now

$$
\forall x \in X, \exists p_{x} \in G, \exists \xi_{x} \in \operatorname{dom}\left(g_{p_{x}}\right)\left(x=g_{p_{x}}\left(\xi_{x}\right)\right),
$$

and then for all $x \in X, h(x)=g_{q_{x}}\left(\xi_{x}\right)$, where $q_{x} \in G$ is such that $\alpha_{q_{x}}$ is minimal with $\xi_{x} \in d o m g_{q_{x}}$. The map $\alpha_{p_{x}} \mapsto \alpha_{q_{x}}$ is regressive on $S_{X}$, and hence we can find $W \subseteq X$, and $\eta<\omega_{1}$ such that $S_{W}$ is stationary and for all $x \in W, \alpha_{q_{x}}=\eta$. Let $q \in G$ be such that $\alpha_{q}=\eta$. Then for all $x \in W, h(x)=g_{q}\left(\xi_{x}\right)$. As $d o m g_{q}$ is countable, there are $V \subseteq W$ and $\xi \in d o m g_{q}$ such that $S_{V}$ is stationary and for all $x \in V, \xi_{x}=\xi$. Then for all $x \in V, x=g_{p_{x}}(\xi)$. Choose $x \neq y$ in $V$, and let $p \in G$ be such that $p \leq p_{x}, p_{y}$. Then $g_{p}(\xi) \geq_{p} x=g_{p_{x}}(\xi), y=g_{p_{y}}(\xi)$. It follows that $x$ and $y$ are compatible, and we get a contradiction. The lemma follows.

Thus in $V[G][H],\left\langle T, \leq_{T}\right\rangle$ is an almost Souslin regressive Kurepa tree with $\kappa$-many branches. This completes the proof of Theorem 2.1.

Remark 2.7. As we will see in the next section, just working in $V[G]$, it is possible to define a subtree $T^{*}$ of $T$ which is an almost Souslin regressive Kurepa tree with $\kappa$-many branches. We gave the above argument for Theorem 2.1 , since it was our original motivation for defining $T^{*}$.

\section{Almost Souslin Kurepa trees in $L$}

In this section, answering Question 2.2, we show that an almost Souslin Kurepa tree exists in $L$. Again as in section 2, we prove something stronger.

Theorem 3.1. If there exists an $\left(\omega_{1}, 1\right)$-morass, then there is an almost Souslin regressive Kurepa tree.

To prove the above theorem, we need some definitions and facts from [15. Let $(\mathbb{P}, \leq)$ be a partial order and $\mathbb{D}=\left\{D_{\alpha}: \alpha<\omega_{2}\right\}$ be a family of open dense subsets of $\mathbb{P}$. For $p \in \mathbb{P}$, let $\operatorname{rlm}(p)=\left\{\alpha<\omega_{2}: p \in D_{\alpha}\right\}$, and for $\alpha<\omega_{2}$ let $\mathbb{P}_{\alpha}=\{p \in \mathbb{P}: \operatorname{rlm}(p) \subseteq \alpha\}$. Also let $\mathbb{P}^{*}=\bigcup_{\alpha<\omega_{1}} \mathbb{P}_{\alpha}$.

Definition 3.2. $\mathbb{D}$ is an $\omega_{1}$-indiscernible family if the following conditions are satisfied:

(1) $\mathbb{P}^{*} \neq \emptyset$, and for all $\alpha<\omega_{1}, \mathbb{P}^{*} \cap D_{\alpha}$ is open dense in $\mathbb{P}^{*}$.

(2) For all $\alpha<\omega_{1},\left(\mathbb{P}_{\alpha}, \leq\right)$ is $\omega_{1}$-closed.

Also for each order-preserving function $f: \alpha \rightarrow \gamma, \alpha<\omega_{1}, \gamma<\omega_{2}$, there is a function $\sigma_{f}: \mathbb{P}_{\alpha} \rightarrow \mathbb{P}_{\gamma}$ such that:

(3) $\sigma_{f}$ is order-preserving.

(4) For all $p \in \mathbb{P}_{\alpha}, r \operatorname{lm}\left(\sigma_{f}(p)\right)=f[r \operatorname{lm}(p)]$. 
(5) If $\beta<\omega_{1}, f|\beta=i d| \beta, f(\beta) \geq \alpha, \gamma<\omega_{1}$ and $p \in \mathbb{P}_{\alpha}$, then $p$ and $\sigma_{f}(p)$ are compatible in $\mathbb{P}^{*}$.

(6) If $f_{1}: \alpha_{1} \rightarrow \alpha_{2}, f_{2}: \alpha_{2} \rightarrow \gamma$ are order-preserving, $\alpha_{1}, \alpha_{2}<\omega_{1}, \gamma<\omega_{2}$, then $\sigma_{f_{2} \circ f_{1}}=\sigma_{f_{2}} \circ \sigma_{f_{1}}$.

We also need the following theorem (see [15, Theorem 1.1.3).

Theorem 3.3. The following are equivalent:

(a) There exists an $\left(\omega_{1}, 1\right)$-morass.

(b) Whenever $\mathbb{P}$ is a partial order and $\mathbb{D}$ is an $\omega_{1}$-indiscernible family of open dense subsets of $\mathbb{P}$, then there is a set $G$ which is $\mathbb{P}$-generic over $\mathbb{D}$. Furthermore $G$ can be chosen to be $\omega_{1}$-complete.

We are now ready to give the proof of Theorem 3.1.

Assume an $\left(\omega_{1}, 1\right)$-morass exists. Let $(\mathbb{P}, \leq)$ be the forcing notion of section 2 , when $\kappa=\omega_{2}$, for adding a regressive Kurepa tree with $\omega_{2}$-many branches. For each $\alpha<\omega_{2}$, let $D_{\alpha}=\left\{p \in \mathbb{P}: \alpha \in\right.$ domg $\left._{p}\right\}$, and let $\mathbb{D}=\left\{D_{\alpha}: \alpha<\omega_{2}\right\}$. Then it is easy to see that for each $p \in \mathbb{P}, r \operatorname{lm}(p)=d^{\circ o m} g_{p}$, for each $\alpha<\omega_{2}, \mathbb{P}_{\alpha}=\{p \in \mathbb{P}$ : $\left.\operatorname{domg}_{p} \subseteq \alpha\right\}$, and $\mathbb{P}^{*}=\mathbb{P}_{\omega_{1}}$.

By Theorem 1.2.1 of $15, \mathbb{D}$ is an $\omega_{1}$-indiscernible family of open dense subsets of $\mathbb{P}$. Thus using Theorem 3.3, there exists $G \subseteq \mathbb{P}$ which is $\mathbb{P}$-generic over $\mathbb{D}$ and is $\omega_{1}$-complete. Define $T, \leq_{T}$ and $f$ exactly as in the proof of Theorem 2.1. By Theorem 1.2.2 of [15], $\left\langle T, \leq_{T}\right\rangle$ is a normal Kurepa tree, and using $f$ it is regressive.

We now define a subtree of $T$ which is an almost Souslin regressive Kurepa tree. Let $S=\left\{\alpha_{p}: p \in G\right\}$ and let $C$ be the set of limit points of $S$. Then $C$ is a club subset of $\omega_{1}$. We first define by induction on $\alpha<\omega_{1}$ a sequence $\left\langle T^{\alpha}: \alpha<\omega_{1}\right\rangle$ of subtrees of $T$ as follows:

- $\alpha=0$ : Let $T^{0}=T$.

- $\alpha=\beta+1:$ Let $T^{\alpha}=T^{\beta}$.

- $\alpha$ is a limit ordinal, $\alpha \notin C$ : Let $T^{\alpha}=\bigcap_{\beta<\alpha} T^{\beta}$.

- $\alpha \in C$ : First let $T^{*}=\bigcap_{\beta<\alpha} T^{\beta}$. Now we define $T^{\alpha}$ as follows:

$-\left(T^{\alpha}\right)_{<\alpha}=\left(T^{*}\right)_{<\alpha}$,

$-\left(T^{\alpha}\right)_{\alpha}=\left\{x \in\left(T^{*}\right)_{\alpha}: \exists \xi<\kappa, \forall p \in G\left(\alpha_{p}<\alpha \wedge \xi \in d o m g_{p} \Rightarrow g_{p}(\xi) \leq_{T} x\right)\right\}$,

- for $\gamma>\alpha,\left(T^{\alpha}\right)_{\gamma}=\left\{x \in\left(T^{*}\right)_{\gamma}: \exists y \in\left(T^{\alpha}\right)_{\alpha}, x \geq_{T} y\right\}$.

Remark 3.4. For $x \in\left(T^{\alpha}\right)_{\alpha}$, the required $\xi$ is unique. Furthermore if $y \in T^{\alpha}$ and $x \leq_{T} y$, then $x \in T^{\alpha}$.

Finally let $T^{*}=\bigcap_{\alpha<\omega_{1}} T^{\alpha}$. Clearly $T^{*}$ is a subtree of $T$ with $\omega_{2}$-many branches, and hence it is a regressive Kurepa tree. We show that it is almost Souslin.

For $\alpha \in C$ we define $g_{\alpha}: \bigcup\left\{\right.$ domg $\left._{p}: p \in G, \alpha_{p}<\alpha\right\} \rightarrow\left(T^{*}\right)_{\alpha}$ as follows: Let $\xi \in$ domg $_{p}$, where $p \in G, \alpha_{p}<\alpha$. Then there is a unique $x \in\left(T^{*}\right)_{\alpha}$ such that

$$
\forall q \in G\left(\alpha_{q}<\alpha \wedge \xi \in \operatorname{domg}_{q} \Rightarrow x \geq_{T} g_{q}(\xi)\right) \text {. }
$$

Let $g_{\alpha}(\xi)=x$. Let us note that $g_{\alpha}$ is a bijection and for $\alpha \in C \cap S, g_{\alpha}=g_{p}$, where $p \in G$ is such that $\alpha_{p}=\alpha$. Next we define a function $h: T^{*} \mid C \rightarrow T^{*}$ as follows: Let $\alpha \in C$ and $x \in\left(T^{*}\right)_{\alpha}$. Then $x=g_{\alpha}(\xi)$ for some $\xi \in d o m g_{\alpha}$. Let $h(x)=g_{\beta}(\xi)$, where $\beta$ is the least ordinal such that for some $p \in G, \beta=\alpha_{p}$, and $\xi \in d o m g_{p}$. Note that $\beta<\alpha$ and $h(x)<_{T} x$. Now as in the proof of Lemma 2.6, we can show that $T^{*}$ is almost Souslin. Hence $T^{*}$ is an almost Souslin regressive Kurepa tree. This completes the proof of Theorem 3.1. 
Remark 3.5. The methods of this paper can be used to get more consistent results about trees. For example we can show that the existence of an almost Souslin Kurepa tree with no Aronszajn subtrees is consistent with $Z F C$ and that such a tree exists in $L$.

The following question from [16 remains open.

Question 3.6. Does there exist a Souslin tree $T$ such that for each $G$ which is $T$-generic over $V, T$ is an almost Souslin Kurepa tree in $V[G]$ ?

\section{REFERENCES}

[1] J. Devlin, S. Shelah, Souslin properties and tree topologies, Proc. Lond. Math. Soc. (3), vol. 39 (1979), 237-252. MR.0548979 (80m:54031)

[2] T. Jech, Non-provability of Souslin's hypothesis, Comment. Math. Univ. Carolinae 8 (1967), 291-305. MR0215729 (35:6564)

[3] T. Jech, Trees, J. Symbolic Logic, vol. 36 (1971), 1-14. MR0284331 (44:1560)

[4] T. Jech, Set theory, 3rd millennium ed., 2003, Springer Monographs in Mathematics. MR.1940513 (2004g:03071)

[5] R. B. Jensen, SH is incompatible with $V=L$, Notices of the American Mathematical Society, vol. 15 (1968), 935.

[6] R. B. Jensen, The fine structure of the constructible hierarchy, Ann. Math. Logic 4 (1972), 229-308; erratum, ibid. 4 (1972), 443. MR0309729 (46:8834)

[7] R. B. Jensen, $\mathrm{SH}$ is compatible with $\mathrm{CH}$ (mimeographed).

[8] B. König, Y. Yoshinobu, Kurepa trees and Namba forcing, in preparation.

[9] S. Shelah, Proper forcing, Lecture Notes in Mathematics, 940. Springer-Verlag, Berlin-New York, 1982. MR0675955 (84h:03002)

[10] J. Silver, The independence of Kurepa's conjecture and two-cardinal conjectures in model theory, 1971 Axiomatic Set Theory (Proc. Sympos. Pure Math., Vol. XIII, Part I, Univ. California, Los Angeles, Calif., 1967), pp. 383-390. Amer. Math. Soc., Providence, R.I. MR0277379 $(43: 3112)$

[11] R. Solovay, S. Tennenbaum, Iterated Cohen's extensions and Souslin's problem, Ann. of Math. (2) 94 (1971), 201-245. MR0294139(45:3212)

[12] D. H. Stewart, M.Sc. Thesis, Bristol, 1966.

[13] S. Tennenbaum, Souslin's problem, Proc. Nat. Acad. Sci. U.S.A. 59 (1968), 60-63. MR.0224456 $(37: 55)$

[14] S. Todorčević, Trees and linearly ordered sets, Handbook of set-theoretic topology, 235-293, North-Holland, Amsterdam, 1984. MR0776625 (86h:54040)

[15] D. Velleman, Morasses, diamond and forcing, Ann. Math. Logic 23 (1982), no. 2-3, 199-281 (1983). MR0701126 (85e:03121)

[16] M. Zakrzewski, Some problems concerning Kurepa trees, J. Symbolic Logic, Vol. 52, No. 3 (1987), 894.

Department of Mathematics, Shahid Bahonar University of Kerman, Kerman, Iran And - School of Mathematics, Institute for Research in Fundamental Sciences (IPM), TEHRAN, IRAN

E-mail address: golshani.m@gmail.com 